\title{
Invited Discussion on: Age- and Gender-Related Variability in Nasal Tip Support
}

\author{
Ashkan Ghavami ${ }^{1}$
}

Received: 22 February 2020/ Accepted: 26 February 2020/Published online: 9 March 2020

(C) Springer Science+Business Media, LLC, part of Springer Nature and International Society of Aesthetic Plastic Surgery 2020

Level of Evidence $V$ This journal requires that authors assign a level of evidence to each article. For a full description of these Evidence-Based Medicine ratings, please refer to the Table of Contents or the online Instructions to Authors www.springer.com/00266.

Nasal tip support and position are critical elements in optimizing rhinoplasty outcomes [1]. It is not uncommon to lose support and, therefore, ideal nasal tip position after a rhinoplasty. The authors have conducted a thoughtful study to more objectively evaluate variations in tip support and strength in an age spectrum of cohorts of ethnically Turkish males and females. Despite the objective use of a Newton caliper, this is a daunting task. Objectifying nasal tip excursion and attributing pressure-resistant variations to age and sex are complicated by numerous factors.

The study found that those between 20 and 29 years of age had statistically higher nasal tip resistance at 2, 3 and $4 \mathrm{~mm}$ displacement when compared to 30-39 and 40-64-year-old cohorts. In addition, males overall had higher tip strength against 3 and $4 \mathrm{~mm}$ displacement compared to females. The limitations of the study were listed but are important to recognize and further delve into. Analyzing tip strength by any method is confounded by a plethora of anatomical interand intra-cohort variations. Within any specific ethnicity, there are still marked variations in cartilage strength and dimension in addition to orientation angles.

With respect to the lower lateral and medial crura, the thickness, length, orientation, width and relationship to

Ashkan Ghavami

ashghavami@yahoo.com

1 Division of Plastic Surgery, Department of Surgery, David Geffen School of Medicine at UCLA, 433 N. Camden Dr. Suite 780, Beverly Hills, CA 90210, USA surroundings are all influential to nasal tip strength. All the preceding traits will determine how the nasal tip is positioned statically as well as dynamically. Its resistance to a posteriorly directed pressure such as with the Newton caliper used in the study will be determined by these anatomical variations which can be significantly different within any cohort. For example, male Turks aged 20-29 can each have variations in: how thick their lower lateral crura are; at what angle they are natively oriented with respect to the anterior septal angle; what their pivot position is relative to the medial crura; and how they are related to the internal lining, ligaments, and outer skin envelope (which itself can have variations in dermal thickness and rigidity). Furthermore, even age-related calcifications can add rigidity in some cartilages so long as the force is not great enough to crack or break the cartilage. Displacement is inherently a dynamic process. These variations make it a challenge to determine tip resistance variations numerically. However, the findings of the study are intuitively what one would expect. That is, males and younger patients would be expected to have stronger tip cartilage as well as less ligamentous laxity.

The biomechanics of the nasal tip tripod cartilages are directly determined by their anatomical characteristics, and improving their resistance to tip ptosis postoperatively requires specific maneuvers that should be employed in all genders and age-groups. This can involve direct treatment of an existing functional or aesthetic insufficiency in tip position and shape or prevention of future unwanted tip ptosis [2]. It is in this that the authors' conclusions hold most value. Understanding that females and older agegroups are prone to loss of tip support and should influence the decision making to reinforce the tripod cartilages via struts and other maneuvers [3]. Surgeons will still need to evaluate individual cartilage and soft tissue components to 
determine whether translocation and/or bilamination is required as well as how much native ligamentous attachments to preserve or re-establish.

Decision making in the medial crura reinforcement, which are often weak and dominated by the lower lateral cartilages, can include a columellar strut graft versus a septal extension graft. The findings of this study only provide a unidirectional assessment of resistance and give further support for technique decision making. The preservation rhinoplasty concept is also intriguing in that the ligaments that are "preserved" may not be that critical in certain patient cohorts who determine native weakness [4]. Or conversely, may be even more critical to preserve if female or above the age of 40 as this study would imply. Perhaps, in the weaker tip groups, ligamentous preservation is not as important as support grafts and struts. Lastly, one must consider that in its native form many nasal tips were not meant to resist a posterior directed force and the spring mechanism of the tripod cartilages may be there to absorb some of the force and protect the deeper septal structures and cranial cavity. Over-strengthening of these cartilages particularly in females for the sake of strength alone is rarely warranted especially in primary rhinoplasty. There is something to be said about softness of the nasal anatomy as long as shape and positional proportions are aesthetically improved.

The conclusions of this study are intuitive and logical for those who routinely perform rhinoplasty in multiple ethnicities and age-groups. While numerical data are not as important to memorize, the observational trends and findings should make the rhinoplasty surgeon more diligent on treating tip support, all-be-it by strut support, re-positioning and/or ligament preservation versus reinforcement, while not being overly concerned about resistance to posterior forces.

\section{Compliance with Ethical Standards}

Conflict of interest The author declares that he has no conflicts of interest to disclose.

Ethical Approval This article does not contain any studies with human participants or animals performed by any of the authors.

Informed Consent For this type of study, informed consent is not required.

\section{References}

1. Janeke JB, Wright WK (1971) Studies on the support of the nasal tip. Arch Otolaryngol 93(5):458-464

2. Ghavami A, Janis JE, Acikel C, Rohrich RJ (2008) Tip shaping in primary rhinoplasty: an algorithmic approach. Plast Reconstr Surg 122:1229

3. Rohrich RJ, Hollier LH, Janis JE, Kim J (2004) Rhinoplasty with advancing age. Plast Reconstr Surg 114:1936-1944

4. Daniel RK, Palhazi P (2018) The nasal ligaments and tip support in rhinoplasty: an anatomical study. Aesthet Surg J 38(4):357-368

Publisher's Note Springer Nature remains neutral with regard to jurisdictional claims in published maps and institutional affiliations. 